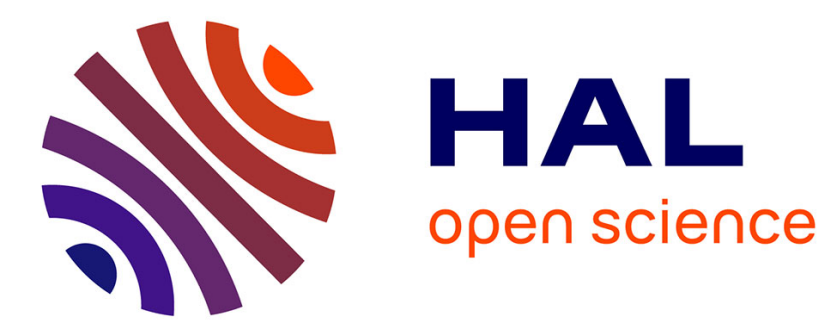

\title{
Mathematical Models of Microwave Ferrites
}

E. Schloemann

\section{To cite this version:}

E. Schloemann. Mathematical Models of Microwave Ferrites. Journal de Physique IV Proceedings, 1997, 07 (C1), pp.C1-433-C1-436. 10.1051/jp4:19971176 . jpa-00254824

\section{HAL Id: jpa-00254824 https://hal.science/jpa-00254824}

Submitted on 1 Jan 1997

HAL is a multi-disciplinary open access archive for the deposit and dissemination of scientific research documents, whether they are published or not. The documents may come from teaching and research institutions in France or abroad, or from public or private research centers.
L'archive ouverte pluridisciplinaire HAL, est destinée au dépôt et à la diffusion de documents scientifiques de niveau recherche, publiés ou non, émanant des établissements d'enseignement et de recherche français ou étrangers, des laboratoires publics ou privés. 


\title{
Mathematical Models of Microwave Ferrites
}

\author{
E.F. Schloemann
}

38 Brook Rd., Weston MA 02193 U.S.A.

\begin{abstract}
Computer-aided design (CAD) of ferrite microwave devices requires an accurate, yet simple mathematical model of the permeability of polycrystalline ferrites. Contrary to earlier mathematical models, the model described in the present paper takes account of the variation of $\mathrm{dc}$ moment with bias field, and incorporates the "low-field loss" observed in ferrites, when magnetized incompletely. Typical input parameters are: frequency, external magnetic bias field, demagnetizing factor, saturation magnetization, remanence ratio, anisotropy field (or equivalent), and two phenomenological damping constants. The calculations are based on the assumption that the polycrystalline ferrite consists of grains having different orientations. In the simplest version of the theory, the easy axes of all grains are all inclined at the same angle to the bias field direction (but are otherwise randomly oriented), and the grains have uniaxial anisotropy. In more sophisticated versions of the theory, the angle between the easy axes and the bias field direction is characterized by a suitable distribution function. Two self-contained FORTRAN subroutines based on these mathematical models and suitable for CAD of ferrite microwave devices are described.
\end{abstract}

\section{INTRODUCTION}

The magnetic response of dc-biased ferrites to electromagnetic fields can generally be characterized by a permeability tensor $\vec{\mu}$. If the direction of the dc-bias field is defined as the z-direction, the non-zero components of this tensor will be $\mu_{x x}, \mu_{x y}, \mu_{y x}, \mu_{y y}$ and $\mu_{z z}$. Calculation of these tensor components based on the generally accepted equations of motion (the Landau-Lifshitz-Gilbert equations) leads to very simple expressions for the dependence of the tensor components on frequency, bias field strength, saturation magnetization, and damping parameters [1-3]. Although these conventional, very simple expressions have been helpful in analyzing and designing ferrite microwave devices, their accuracy leaves much room for improvement. The design of ferrite microwave devices has therefore largely been empirical, with theoretical analysis providing only limited guidance.

The conventional theoretical expressions for the microwave permeability of ferrites have two major shortcomings: a) they fail to take account of the variation of the dc magnetic moment with dc bias field strength, and b) they do not incorporate the "low-field loss" generally observed in ferrites that are magnetized incompletely. These shortcomings are significant, because very often the optimum performance of practical devices, such as circulators, is realized at low dc bias fields, i.e. under conditions when the deficiencies are most pronounced. The objective of the present work is therefore to develop a more accurate, but still relatively simple theoretical model of microwave ferrites for use in computer-aided design (CAD).

An approximate theory of low-field loss in partially magnetized ferrites has previously been published [4], and was found to be in qualitative agreement with experimental observations[5]. In this earlier theory the variation of $\mathrm{dc}$ magnetic moment with $\mathrm{dc}$ bias field is neglected by way of approximation ("constant- $\mathrm{M}_{\mathrm{Z}}$ " model). Even though this approximation may be justified under some conditions, it can not be expected to be generally valid, and serve as a reliable basis of CAD of ferrite microwave devices.

The "variable- $\mathrm{M}_{\mathrm{Z}}$ " model described in the present paper applies to polycrystalline ferrites, and is based on the assumption that the single-crystal grains of the material have different orientations. In the simplest version of the theory, the grains are assumed to have uniaxial anisotropy, and the easy axes of all grains are assumed to be inclined at the same angle to the bias field direction (but are otherwise randomly oriented). In a more realistic version of the theory, the angle between the grain easy axes and the bias field direction is assumed to be given by a plausible distribution function, and the grains are again assumed to have uniaxial anisotropy. A generalization to materials with cubic anisotropy is discussed briefly.

For a fully magnetized sample of ellipsoidal shape, the microwave permeability is expected to be a function of the internal magnetic field $H_{\text {int }}$, which is related to the external magnetic field by

$$
H_{\mathrm{int}}=H_{\text {ext }}-4 \pi M N_{2}
$$

where $M$ is the dc magnetization, and $N_{z}$ is the demagnetizing factor for the z-direction, in which the field is applied. In order to be most useful for $\mathrm{CAD}$ a mathematical model of microwave ferrites should be formulated in terms of the externally applied field, rather than the internal field, because a) the external field, rather than the internal field can be directly measured and adjusted, and b) for incompletely magnetized ferrites, the low-field loss is a function of dc magnetization that cannot easily be expressed in terms of the internal magnetic field, but it can be expressed in terms of the external field. 


\section{SIMPLE MODEL.}

\subsection{Moment calculation}

The calculations summarized in Sections 2.1 and 2.2 are based on the assumption that the easy axes of all grains deviate from the $\mathrm{dc}$ field direction by the same amount, being otherwise randomly oriented. Fig. 1 illustrates the conventions used in these sections, in particular in the calculation of the dc magnetization. The local easy axis of a given crystallite is assumed to deviate from the dc field direction by a polar angle $\theta_{0}$ and to have azimuth angle $\phi_{0}$. An arbitrary magnetization vector is shown as having a polar angle $\theta$ and azimuth $\phi$. With the angle $\psi$ in Fig.1 given by Eq.(2.1) in Fig.2, the uniaxial crystalline anisotropy contributes to the magnetic energy a term proportional to $\sin ^{2} \psi$. The other significant terms in the energy are the Zeeman energy in the externally applied field and the demagnetization energy. With respect to the latter, it should be noted that the sample as a whole has only a $x$-component of magnetization, even though individual grains generally have $x$ - and $y$-components. The total magnetic energy per unit volume (in Gaussian units) is therefore given by Eq.(2.2) of Fig.2, where $H_{a}$ is the anisotropy field.

The equilibrium direction of the grain magnetization is defined by the minimum of the magnetic energy function (2.2). A minimum with respect to $\phi$ is realized for $\phi=\phi_{0}$. Thus the energy function simplifies to the expression given in Eq.(2.3). The derivative of $\mathrm{E}$ with respect to $\theta$ can be written in terms of the external field in the form (2.4), or alternatively in terms of the internal field in the form (2.5), where $H_{\text {int }}$ is given by Eq.(1) with $M=M_{s} \cos \theta$. The equilibrium condition $\partial E / \partial \theta=0$ can be solved explicitly in the form (2.6), which shows $H_{\text {int }}$ as an explicit function of the magnetization direction $\theta$ and the misalignment angle $\theta_{0}$. To solve Eq.(2.4) for $\theta$ given $\theta_{0}, H_{e x t}, H_{a}, M_{s}$ and $N_{z}$, Newton's method can be used.

A computer program has been developed that calculates the dc magnetization as part of a procedure that also calculates the microwave permeability. the input parameters required for the dc moment calculation are $H_{e x t}, H_{\alpha}, M_{s}, N_{z}$ and the normalized remanent magnetization $R_{m}$. In the present case (equal misalignment angles for all grains)

$$
R_{m}=\cos \theta_{0}
$$

Figure 3 shows the calculated dependence of normalized magnetization on applied magnetic field, assuming 5 values of $R_{m}$ In this graph, and similar graphs that follow, all magnetic field strengths are expressed in frequency units (typically $\mathrm{GHz}$ ). Thus $f_{m}$ is the saturation magnetization $\left(4 \pi M_{s}\right.$ in the Gaussian system) and $f_{a}$ is the anisotropy field. The value of $f_{m}$ assumed in Fig.3 corresponds to yttrium iron garnet (YIG), the assumed value for $f_{a}$ is about 4 times higher than appropriate for YIG. It may be seen that the $M$ ys $H_{e x t}$ curves generally consist of two branches. On the low-field branch $M$ increases linearly with $H_{\text {ext }}$, whereas on the high-field branch $\mathrm{M}$ gradually approaches $M_{s}$ with increasing $H_{e x t}$. The two branches are joint at the "kink field"

$$
H_{e x k k}=f_{m} R_{m} N_{z}
$$

\subsection{Microwave permeability calculation}

The microwave permeability is first calculated for an isolated grain in a coordinate system that is aligned

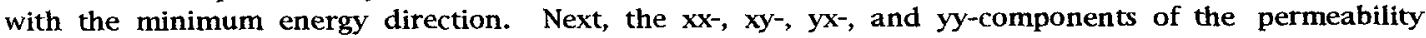
tensor in a coordinate system aligned with the bias field direction ( $\mathrm{z}$-axis ) are calculated, taking into account the random distribution of the azimuth angles of the local easy axes. This calculation is first carried out for $H_{e x t} \geq H_{e x t k}$, then for $H_{e x t} \leq H_{e x t k}$.

The equations of motion for $H_{e x t} \geq H_{e x t k}$ can be derived from the magnetic energy density given in Eq.(2.2) by well-known methods. The microwave permeability at bias fields smaller than the kink field ( $H_{e x t} \leq H_{e x t k}$ ) can be deduced from theoretical expressions for the case of zero misalignment, which have been derived in a previous publication [4]. These expressions are based on the physical mechanism first proposed by Polder and Smit[6] and take account of other, more recent work [7,8,9]. In order to generalize the theory to the present case, it is necessary to recognize that, by assumption, all grains are oriented at the same angle $\theta_{0}$ to the external bias field, the internal field being zero.

In the derivation of the expressions for the microwave permeability, the effect of dissipation loss is at first neglected. To remove this restriction, we take account of a suitable loss torque in the equations of motion. For ferro- and ferrimagnetic materials this loss torque must be such that the length of the local, microscopic magnetization vector remains constant, as implied in the Landau-Lifshitz-Gilbert 
equations of motion. Analysis shows that the effect of damping can formally be taken into account by simply replacing the magnetic field $\gamma H_{z}$ by a complex variable $\gamma H_{z}+j \omega \alpha$, where $\alpha$ is a damping parameter and $\omega$ the frequency.

The loss term derived from the Landau-Lifshitz-Gilbert equation implies that the intrinsic line width is proportional to frequency. Experimental data on many materials indicate that such a term is indeed present, but that a constant term also appears to be necessary for adequate agreement. In the present work, such a frequency independent term is taken into account. All resonance frequencies $\omega_{\text {res }}$ are replaced by complex variables, according to

$$
\omega_{r e s} \rightarrow \omega_{r e s}+j\left(\omega \alpha+\Delta \omega_{0} / 2\right)
$$

Here $\Delta \omega_{0}$ is the intrinsic line width at low frequencies (formally, extrapolated to $\omega=0$ ).

The loss parameters $\alpha$ and $\Delta \omega_{0}$ for use in CAD of ferrite microwave devices must be deduced from the intrinsic line width inferred from off-resonance measurements. In the literature the somewhat misleading term "effective line width" is often used for this parameter. For polycrystals the directly observed width of the resonance line is usually much larger than the intrinsic line width, due to inhomogeneities, such as caused by crystalline anisotropy, as further discussed in Section 3.

The mathematical model described above has been incorporated in a FORTRAN program as a subroutine that will first calculate the dc magnetization and then the microwave permeability components. The program is structured such that it can easily be inserted in any CAD program as a self-contained unit. For covenience all input parameters are either dimensionless, such as $N_{z}, R_{m}, \alpha$, or quantities that can be expressed in frequency units (typically in GHz). Fig. 4 shows an example, in which $\mu^{\prime \prime}$ (imaginary part of the diagonal component of the permeability tensor) is plotted as functions of signal frequency, with $H_{e x t}$ as a parameter and fixed values of $f_{m}, f_{a}, \Delta f_{0}, \alpha, N_{z}$ and $R_{m}$. The chosen values are approximately correct for YG and typical circulator designs. A graph of $\mu^{\prime \prime}$ vs external magnetic field, with signal frequency $f$ as parameter is shown in Fig 5. The "kink field" in both examples is $2.8 \mathrm{GHz}$, as manifested in the sharp break in the curves, especially for low signal frequencies.

\section{MORE ACCURATE MODELS}

The assumption, made in the previous section, that the misalignment angle is the same for all grains may be an oversimplification. To address this concern, the theory described above can be extended relatively easily to materials that are characterized by a distribution of misalignment angles. In the present section we assume that the grains have uniaxial anisotropy, and that their easy axes are distributed with uniform density over a circular region around a reference direction, which coincides with the bias field direction. The implications of this "uniform polecap" model for the magnetization curves observed in polycrystalline hexagonal ferrites have been discussed previously [10].

An example of a microwave permeability component calculated assuming a distribution of misalignment angles is shown in Fig.6, which may be compared with Fig.4, because it is based on the same numerical parameters. As may be expected, the calculated curves are similar. The numerical assumptions used in Fig. 4 and 6 correspond approximately to YIG, a material with an exceptionally low anisotropy field. Even for this material some significant differences between Fig. 4 and 6 may be noted: a) The lowfield loss at intermediate bias fields is larger when misalignment is distributed (compare curves for $H_{\text {ext }}$ $=3 \mathrm{GHz}$ ), and $\mathrm{b}$ ) the resonance peaks that occur for $H_{\text {ext }}>3 \mathrm{GHz}$ are much broader and reduced in height. For materials with a larger anisotropy field these two trends will be even more pronounced.

\section{DISCUSSION}

It may be questioned whether the mathematical models described here, being based on the assumption of uniaxial anisotropy, can be applied to the spinel- and garnet-type ferrites, which have cubic crystal symmetry. Admittedly, the theory can only be a rough approximation; but it is not without merit, because the directional dependence of the anisotropy energy in cubic crystals in the vicinity of the easy axes can be approximated by a uniaxial term. Thus the models can be applied to materials with cubic crystal structure, provided that the relevant material parameter $H_{a}$ is chosen appropriately.

In their present form, the mathematical models described here do not take account of the potential presence of pores or non-magnetic phases in the materials. This may be a significant shortcoming. It is to be hoped that future work along the lines suggested in the present paper will ultimately overcome this limitation. 


\section{References}

[1] Polder D. , Phil. Mag.. .40 (1949) 99-115

[2] Rodrigue P. G. , Proc. IEEE, 76 (1988) 121-137

[3] Lax B. and Button K. J. , Microwave Ferrites and Ferrimagnetics, (McGraw-Hill Book Comp.,New York ,1962), p. 150-167

[4] Schloemann E. , IEEE Trans. MAG-28, (1992) 3300-3302

[5] Blight R. E. , and Schloemann E. , IEEE Trans. MAG-28 (1992) 3291-3293

[6] Polder D. and Smit J. , Rev. Mod. Phys. 25 (1953) 89-90

[7] Schloemann E., J. Appl. Phys., 41 (1970) 204-214

[8] Schloemann E., J. Physique. 32 (1971) p. C1-443 to C1-451

[9] Bouchaud J. P. and Zerah P. J. , Phys. Rev. Lett. 63 (1989) 1000-1003

[10] Schloemann E. , IEEE Trans. MAG-3 1 (1995) 3482-3484

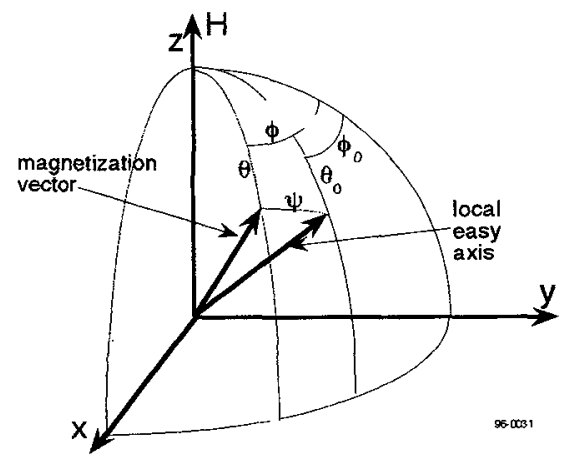

Fig. 1 Conventions used in calculation of dc magnetization

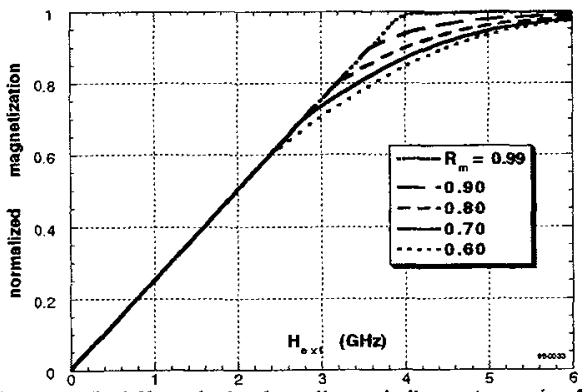

Fig. 3 Normalized Magnetization for uniform misalignment, assoming $t_{m}=5$ GHb $f_{s}=1 \mathrm{GHz}, N_{z}=0.8$ and 5 values of normalized remanent magnetization $R_{m}$

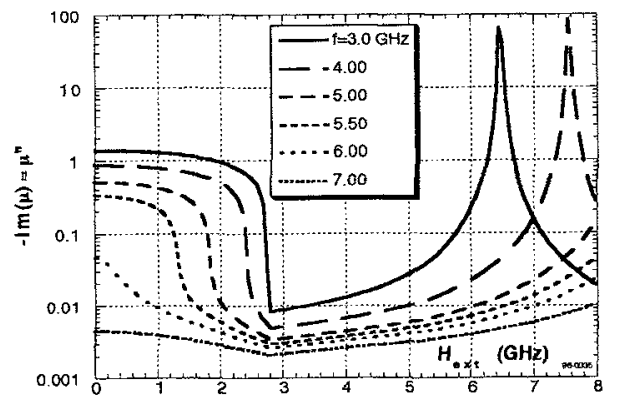

Fig. $5 \mu^{n}$ for $f_{m}=5.0, f_{s}=1.0,8 f_{0}=.01 \mathrm{GHz}, \infty=.003, N_{z}=0.8, R_{m}=0.7$

$$
\begin{aligned}
& \text { Magnetic Energy for uniaxial crystallites } \\
& \cos \psi=\cos \theta_{0} \cos \theta+\sin \theta_{0} \sin \theta \cos \left(\phi_{0}-\phi\right) \\
& E=M_{s}\left[\frac{1}{2} H_{a}\left(1-\cos ^{2} \psi\right)-H_{e x t} \cos \theta+2 \pi M_{s} N_{z} \cos ^{2} \theta\right] \\
& \text { Conditions for minimum Energy } \\
& \qquad \phi=\phi_{0} \rightarrow \cos \psi=\cos \left(\theta_{0}-\theta\right) \\
& E=M_{s}\left[\frac{1}{2} H_{a} \sin ^{2}\left(\theta_{0}-\theta\right)-H_{e x t} \cos \theta+2 \pi M_{s} N_{z} \cos ^{2} \theta\right] \\
& \frac{\partial E}{\partial \theta}=M_{s}\left[-\frac{1}{2} H_{a} \sin \left(2\left(\theta_{0}-\theta\right)\right)+H_{e x t} \sin \theta-4 \pi M_{s} N_{z} \cos \theta \sin \theta\right] \\
& \qquad=M_{s}\left[-\frac{1}{2} H_{a} \sin \left(2\left(\theta_{0}-\theta\right)\right)+H_{\mathrm{int}} \sin \theta\right] \\
& \frac{\Phi}{\partial \theta}=0 \quad \rightarrow \quad \frac{H_{\mathrm{int}}}{H_{a}}=\frac{\sin \left(2\left(\theta_{0}-\theta\right)\right)}{2 \sin (\theta)}
\end{aligned}
$$

Fig. 2 Equations for DC Moment Calculation

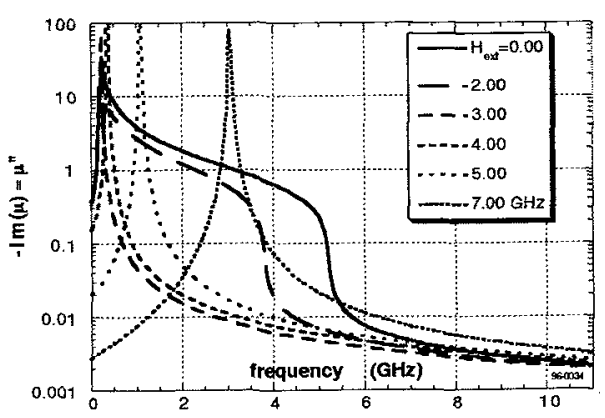

Fig. $4 \mu^{n}$ for $f_{m}=5.0, f_{\alpha}=.224, \delta f_{0}=.01 \mathrm{GHz}, \alpha=.005, \quad N_{z}=0.8, R_{m}=0$

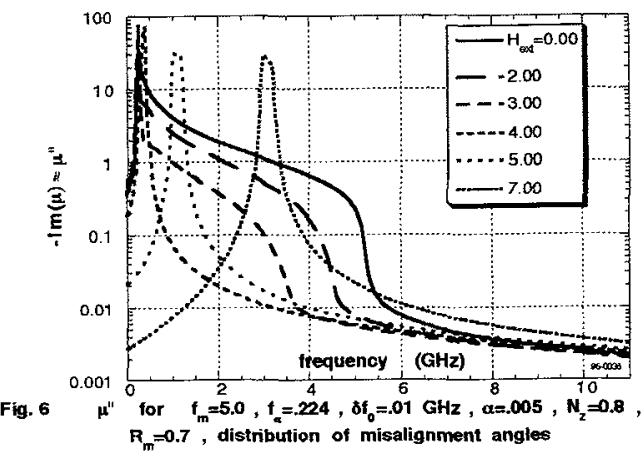

